\title{
Ecología trófica de Girella laevifrons (Pisces: Kyphosidae) en zonas intermareales rocosas del norte de Chile afectadas y no afectadas por contaminantes derivados de la minería del cobre
}

\author{
Trophic ecology of Girella laevifrons (Pisces: Kyphosidae) in rocky intertidal zones \\ of northern Chile affected an non-affected by copper mine tailings
}

JOSE MIGUEL FARIÑA', MARCELA ALDANA, FERNANDO OGALDE \& F. PATRICIO OJEDA

Departamento de Ecología, Facultad de Ciencias Biológicas, Pontificia Universidad Católica de Chile, C.P. 6513677, Santiago, Chile, e-mail: 'ejfarina@genes.bio.puc.cl

\begin{abstract}
RESUMEN
La zonas intermareales de localidades de la costa norte de Chile afectadas por contaminantes derivados de la minería del cobre, presentan una diversidad de especies sésiles menor que las localidades no afectadas. Girella laevifrons es un pez altamente representado en zonas intermareales rocosas del norte de Chile. En el presente trabajo se analiza y compara la ecología trófica de esta especie, en relación con las diferencias en la composición de especies sésiles de sitios contaminados y no contaminados. Los resultados muestran diferencias significativas en la composición trófica de los ejemplares presentes en los sitios afectados y no afectados. Dentro de los sitios afectados, se observan diferencias en los tamaños y en las concentraciones de cobre corporales, las cuales pueden estar relacionadas directamente con la disponibilidad de alimento e indirectamente con interacciones con otros organismos intermareales.
\end{abstract}

Palabras clave: peces intermareales, Girella laevifrons, efluentes de cobre, herbivoría.

\begin{abstract}
The intertidal zone at several sites of the northern Chilean coast affected by copper mine tailings, shows a lower diversity of sessile species than the non-affected ones. Girella laevifrons is a fish highly represented in rocky intertidal shores of these sites. In this study we analized and compared, the dietary composition of this species and its relation with the intertidal sessile assemblages of contaminated and non-contaminated sites. The results show significant differences in the dietary composition of the fishes occurring in contaminated and non-contaminated sites. Within the contaminated sites, the differences in body sizes and in the body concentrations of copper could be related directly to the food availability and indirectly to the interactions with other intertidal species.
\end{abstract}

Key words: intertidal fish, Girella laevifrons, copper mine tailings, herbivory.

\section{INTRODUCCION}

Una serie de localidades de la costa norte de Chile han sido afectadas por la descarga de contaminantes derivados de la minería del cobre (Castilla 1975, Ingeniería y Geotécnica LTDA 1990, Miethke et al. 1999', Vasquez et al. 1999). A pesar de esto, la totalidad de los estudios referentes al efecto de este tipo de contaminantes sobre comunidades del intermareal rocoso han sido desarrollados en sitios ubicados en las cercanías de la ciudad de Chañaral, afectada por la descarga de relaves provenientes de la Mina El Salvador. En estos estudios, se ha descrito que los principales cambios que se producen en las comunidades son una drástica disminución en el número de especies, acompañada por un aumento en la representación espacial de algas verdes efímeras (Castilla \& Nealler 1978). La disminución en la diversidad se debe a la ausencia de grupos impor-

\footnotetext{
' Miethke S, JC Castilla, MC Espoz \& D Oliva (1992) Impacto ambiental por relaves de cobre en comunidades intermareales rocosas de la III Región. XII Jornadas de Ciencias del Mar, Santiago, Chile: 76.
} 
TABLA 1

Lista de especies sésiles observadas en los sitios de estudio obtenida a partir de censos cualitativos (Palito ${ }^{1}$ y cuantitativos (Bandurrias y Santo Domingo ${ }^{2}$ )

List of sesile species observed at the study sites obtained from qualitative (Palito ${ }^{1}$ ) and quantitaive (Bandurrias y Santo Domingo ${ }^{2}$ ) surveys

\begin{tabular}{|c|c|c|c|}
\hline & Palito & Bandurrias & Santo Domingo \\
\hline \multicolumn{4}{|l|}{ Taxon } \\
\hline Enteromorpha compressa & $\mathrm{X}$ & $\mathrm{x}$ & $\mathrm{x}$ \\
\hline \multicolumn{4}{|l|}{ Enteromorpha prolifera } \\
\hline Ulva lactuca & $\mathrm{X}$ & $\mathrm{x}$ & $\mathrm{x}$ \\
\hline Chaetomorpha linum & & $\mathrm{x}$ & \\
\hline Ectocarpus confervoides & $\mathrm{X}$ & $\mathrm{x}$ & $\mathrm{x}$ \\
\hline Ralfsia expansa & $\mathrm{X}$ & $\mathrm{X}$ & $\mathrm{x}$ \\
\hline Colpomenia phaeodactyla & & $\mathrm{X}$ & \\
\hline Colpomenia sinuosa & & $\mathrm{x}$ & \\
\hline Petalonia fascia & & $\mathrm{x}$ & \\
\hline Scytosiphon lomentaria & $\mathrm{X}$ & $\mathrm{X}$ & $\mathrm{x}$ \\
\hline Glosophora kunthii & & $\mathrm{x}$ & \\
\hline Porphyra columbina & & $\mathrm{x}$ & \\
\hline Gelidium chilense & & $\mathrm{x}$ & \\
\hline Gelidium lingulatum & & $\mathrm{x}$ & \\
\hline Corallina officinalis & & $\mathrm{X}$ & \\
\hline Ceramium rubrum & & $\mathrm{X}$ & \\
\hline Hildenbrandtia lecannellieri & $\mathrm{X}$ & $\mathrm{X}$ & $\mathrm{X}$ \\
\hline Roja Incrustante & & $\mathrm{x}$ & \\
\hline Diatomeas & & $\mathrm{x}$ & \\
\hline Anthothoe chilensis & & $\mathrm{x}$ & \\
\hline Phymactis clematis & $\mathrm{x}$ & $\mathrm{x}$ & $\mathrm{X}$ \\
\hline Ascidia Colonial & & $\mathrm{X}$ & \\
\hline Sabellaridae & & $\mathrm{x}$ & \\
\hline Chtamalus scabrosus & & $\mathrm{x}$ & \\
\hline Jehlius cirratus & & $\mathrm{x}$ & \\
\hline Balanus laevis & & $\mathrm{x}$ & \\
\hline Austromegabalanus psittacus & $\mathrm{x}$ & $\mathrm{x}$ & \\
\hline Semimytilus algosus & & $\mathrm{x}$ & \\
\hline Perumytilus purpuratus & $\mathrm{x}$ & $\mathrm{X}$ & $\mathrm{X}$ \\
\hline Pyura chilensis & & $\mathrm{X}$ & \\
\hline TOTAL & 9 & 29 & 8 \\
\hline
\end{tabular}

1Extraida de Castilla (1995)

${ }^{2}$ Extraida de Fariña $(1997)^{2}$

tantes de especies sésiles intermareales (ver Tabla 1) tales como los filtradores (i.e., crustáceos cirripedios y molúscos bivalvos) y las algas rojas (tanto foliosas como incrustantes). El aumento en la representación espacial de algas verdes se debe a la monopolización del substrato por parte del alga Enteromorpha compressa (Linnaeus) (Castilla 1995).

A pesar de los drásticos cambios en el paisaje de la zona intermareal asociados a la presencia de contaminantes derivados de la minería del cobre, en los sitios contaminados es posible observar a una serie de especies que consumen organismos intermareales sésiles, tales como peces (Girella laevifrons), jaibas (Leptograpsus variegattus, Grapsus grapsus) y aves (Cinclodes nigrofumosus)
(JM Fariña, comunicación personal). Hasta el momento, no se han desarrollado estudios que den cuenta comparativamente de la ecología trófica de estas especies en sitios afectados y no afectados por la contaminación derivada de la minería del cobre.

Girella laevifrons (Tschudi) es un pez de amplia representación en zonas intermareales y submareales someras de la costa centro norte de Chile (Stephien 1990, Varas \& Ojeda 1990, Muñoz \& Ojeda 1997). Una serie de estudios desarrollados en la zona central de Chile describen a esta especie como omnívora con una dieta compuesta principalmente por algas verdes, moluscos bivalvos y anfípodos (Varas \& Ojeda 1990, Muñoz \& Ojeda 1997). En base a esto, es esperable que 


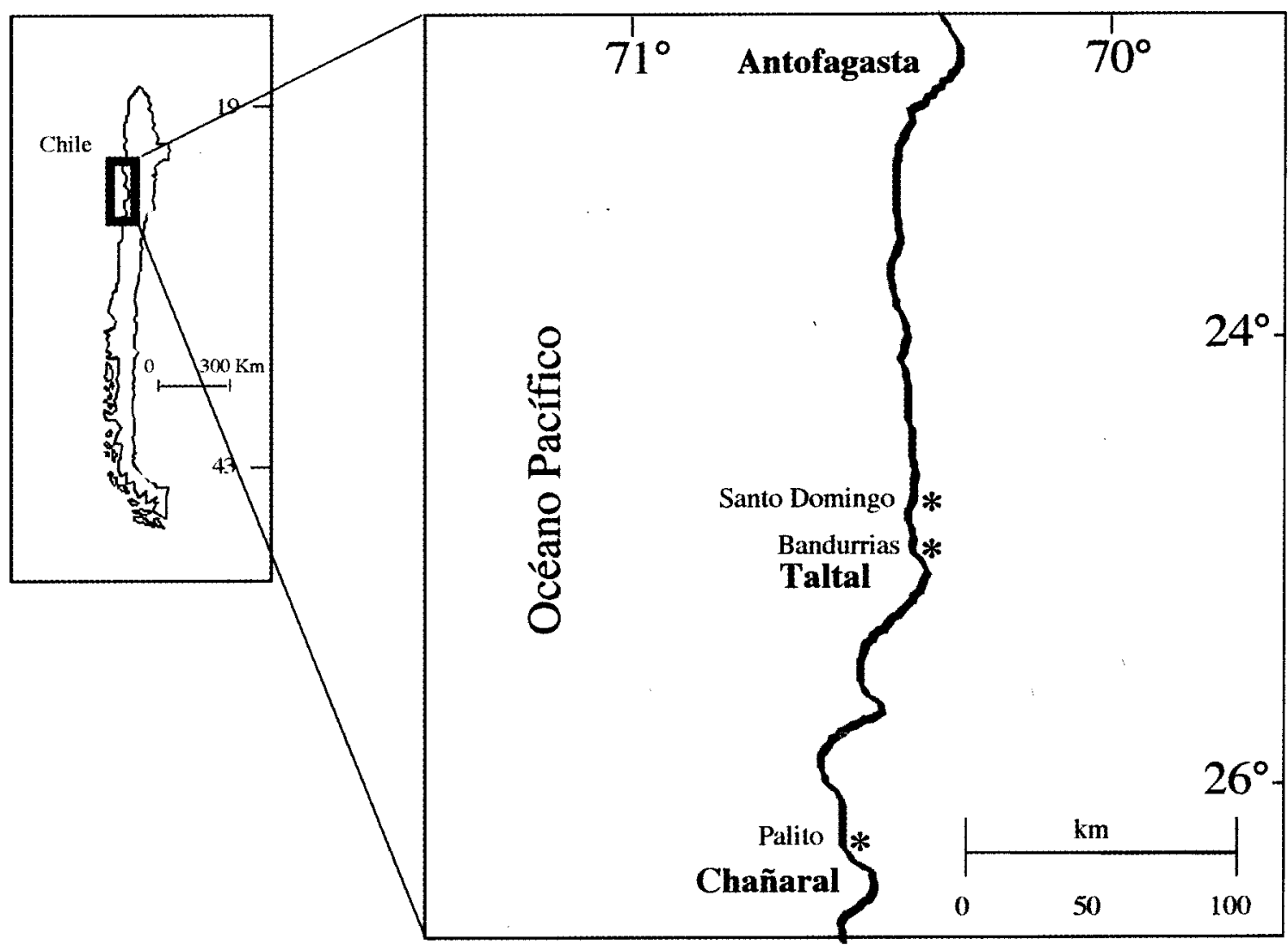

Fig. 1. Localización de los sitios de muestreo (W).

Location of sampling sites (W).

los cambios en la composición de especies intermareales sésiles, que ocurren en los sitios afectados por la contaminación, determinen cambios importantes en la dieta de $G$. laevifrons.

El objetivo del presente trabajo fue analizar y comparar la ecología trófica de G. laeviforns en zonas intermareales rocosas del norte de Chile, afectadas y no afectadas por la presencia de contaminantes derivados de la minería del cobre.

\section{MATERIALES Y METODOS}

El estudio se llevó a cabo en los sitios de Santo Domingo (25 $5^{\circ} 10^{\prime} 45^{\prime}$ ' $\mathrm{S}, 70^{\circ} 27^{\prime} 15^{\prime}$ ' $\mathrm{W}$ ), Bandurrias $\left(25^{\circ} 11^{\prime} 5^{\prime}\right.$ ' $\left.\mathrm{S}, 70^{\circ} 26^{\prime} 25^{\prime}, \mathrm{W}\right)$ y Palito $\left(26^{\circ}\right.$ $17^{\prime} 47^{\prime}$ ' S, 7040'24', W) (Fig. 1). Santo Domingo está ubicado a $40 \mathrm{~km}$. al norte de la cuidad de Taltal. La zona intermareal de esta localidad ha sido afectada desde 1970 por la descarga de relaves de cobre, provenientes la planta de la Minera Punta Grande. Bandurrias está ubicado a $24 \mathrm{~km}$ al norte de la cuidad de Taltal y su zona intermareal no ha sido afectada por relaves de cobre. Palito está ubicado a $8 \mathrm{~km}$ al norte de la cuidad de Chañaral y su zona intermareal ha sido afectada desde 1975 por la descarga de relaves de cobre provenientes de la Mina EL Salvador.

Las tres localidades presentan una zona intermareal rocosa expuesta al oleaje dentro de la cual se alternan zonas de bolones y plataformas de substrato emergente. En las zonas de substrato emergente de cada localidad se eligieron tres plataformas (de orientación y pendiente similares) que presentaran pozas de marea. La composición y abundancia de los organismos sésiles presentes en la zona intermareal rocosa de Santo Domingo y Bandurrias ha sido descrita por Fariña (Fariña $1997^{2}$ ). De igual forma, una serie de trabajos han descrito los patrones de composición y abundancia de especies sésiles presentes en la zona intermareal rocosa de Palito (Castilla 1995, Castilla \& Nealler 1978, ver Tabla 1).

En cada sitio, en Septiembre, Octubre y Diciembre de 1998, se colectó la totalidad de los ejemplares de Girella laevifrons presentes en 3

${ }^{2}$ Fariña JM (1997) Estructura de comunidades intermareales de la II región afectadas por contaminantes derivados de la minería del cobre. XL Reunión de la Sociedad de Biología de Chile, Pucon, Chile: 78. 
pozas intermareales. Debido a que durante estos meses ocurre el reclutamiento de esta especie en la zona intermareal, es el periodo en el cual es posible encontrar las mayores abundancias y rangos de tamaño (Varas \& Ojeda 1990). La colecta se realizó en pozas de volúmenes similares $(5 \pm$ $0,5 \mathrm{~m}^{3}$ ) ubicadas en la zona intermareal baja, utilizando el ictiocida Rotenona. Cada ejemplar fue capturado usando redes de mano y congelado para los análisis en laboratorio. En las pozas de cada sitio se registró, mediante la fotografía de 10 cuadrantes de $0,2 \times 0,2 \mathrm{~m}$, la cobertura de especies sésiles y la abundancia de las especies móviles presentes en las paredes y el fondo rocoso. Para la identificación de estas especies se tomaron muestras de las especies de algas más abundantes en cada uno de los cuadrantes (i.e., Enteromorpha compressa en los sitios contaminados y Ulva sp. en Bandurrias). Adicionalmente, para determinar los niveles de metales pesados presentes en cada sitio, en cada fecha de muestro se colectaron 2 muestras de $11 \mathrm{del}$ agua de cada poza intermareal.

\section{Análisis de laboratorio}

En el laboratorio los ejemplares capturados fueron sexados, pesados y medidos extrayéndoseles el tubo digestivo, cuyo contenido fue analizado al mas bajo nivel taxonómico posible. Se determinó el peso de cada ítem dietario y su frecuencia en el total ejemplares analizados.

La cobertura de las especies sésiles registradas en los cuadrantes, fue estimada a través de la disposición de una grilla con 100 puntos de intersección sobre cada foto. El porcentaje de cobertura fue determinado por la suma del total de puntos de intersección que cada especie ocupó en cada cuadrante.

La concentración de metales pesados en el agua de mar se realizó a través de la técnica de denudación de potenciométrica de electrodos en solución estacionaria utilizando un radiometro computarizado. Para cada sitio se eligió aleatoriamente a 6 ejemplares de $G$. laevifrons, los cuales fueron secados y molidos para analizar el contenido de cobre total de sus tejidos. Asimismo, las muestras de algas verdes colectadas en cada localidad fueron secadas y molidas para realizar el mismo tipo de análisis. Las concentraciones de cobre de los tejidos vegetales y animales se determinaron en un espectrofotómetro de absorción atómica (GBC 909 PBT). Todos los análisis de concentración de metales se realizaron siguiendo las técnicas descritas por Correa et al. (1996).

\section{Análisis estadísticos}

Las diferencias en peso y frecuencia de ocurrencia de los ítems que componen la dieta de los ejemplares capturados en cada sitio, fueron analizadas a través de la prueba de correlación de rangos de Spearman (Siegel \& Castellan 1988).

Las diferencias en la cobertura y abundancia de las especies presentes en las paredes y fondo de las pozas muestreadas fueron analizadas usando un modelo aleatorio de análisis de varianza de una vía (considerando a los sitios como único factor de tres niveles). En el caso de las coberturas, previo al análisis los datos fueron transformados usando el arcoseno de la raíz cuadrada de los datos.

Tomando en cuenta de que el muestreo se realizó dentro de una misma estación (verano), elegida por le ocurrencia de eventos de reclutamiento intermareal de $G$. laevifrons, se agrupó a los ejemplares capturados en cada sitio durante las 3 fechas de muestreo. Las diferencias en el tamaño y peso promedio de los ejemplares fueron analizadas a través de modelos aleatorios de análisis de varianza de una vía (considerando a los sitios como único factor con tres niveles). La relación entre tamaño corporal y peso de los ejemplares capturados en cada sitio se realizó a través de análisis de regresión sobre los datos transformados logarítimicamente $\left(\log _{\mathrm{e}}\right)$.

Las diferencias en la concentración de metales pesados presentes en el agua de mar y en los tejidos animales y vegetales de los organismos colectados en cada sitio, fueron analizadas utilizando modelos aleatorios de análisis de varianza de una vía (considerando a los sitios como único factor con tres niveles). Para cada análisis de varianza, se realizaron contrastes entre los promedios usando la prueba de Student-NewmanKeuls (SNK, Underwood 1997).

En cada análisis paramétrico, la normalidad de los datos fue verificada utilizando métodos gráfi$\cos$, la homogenidad de varianzas fue verificada utilizando la prueba de Levene y la independencia de los datos fue verificada utilizando la prueba de Durbin-Watson (Wilkinson et al. 1996).

\section{RESULTADOS}

\section{Análisis dietario}

En un total de 9 pozas muestreadas ( 3 por sitio), se capturaron 110 ejemplares de Girella laevifrons. Se identificaron 20 ítems presa en los tractos digestivos de estos ejemplares (Tabla 2). En Bandurrias se observó la mayor diversidad de 
ECOLOGIA TROFICA DE G. laevifrons

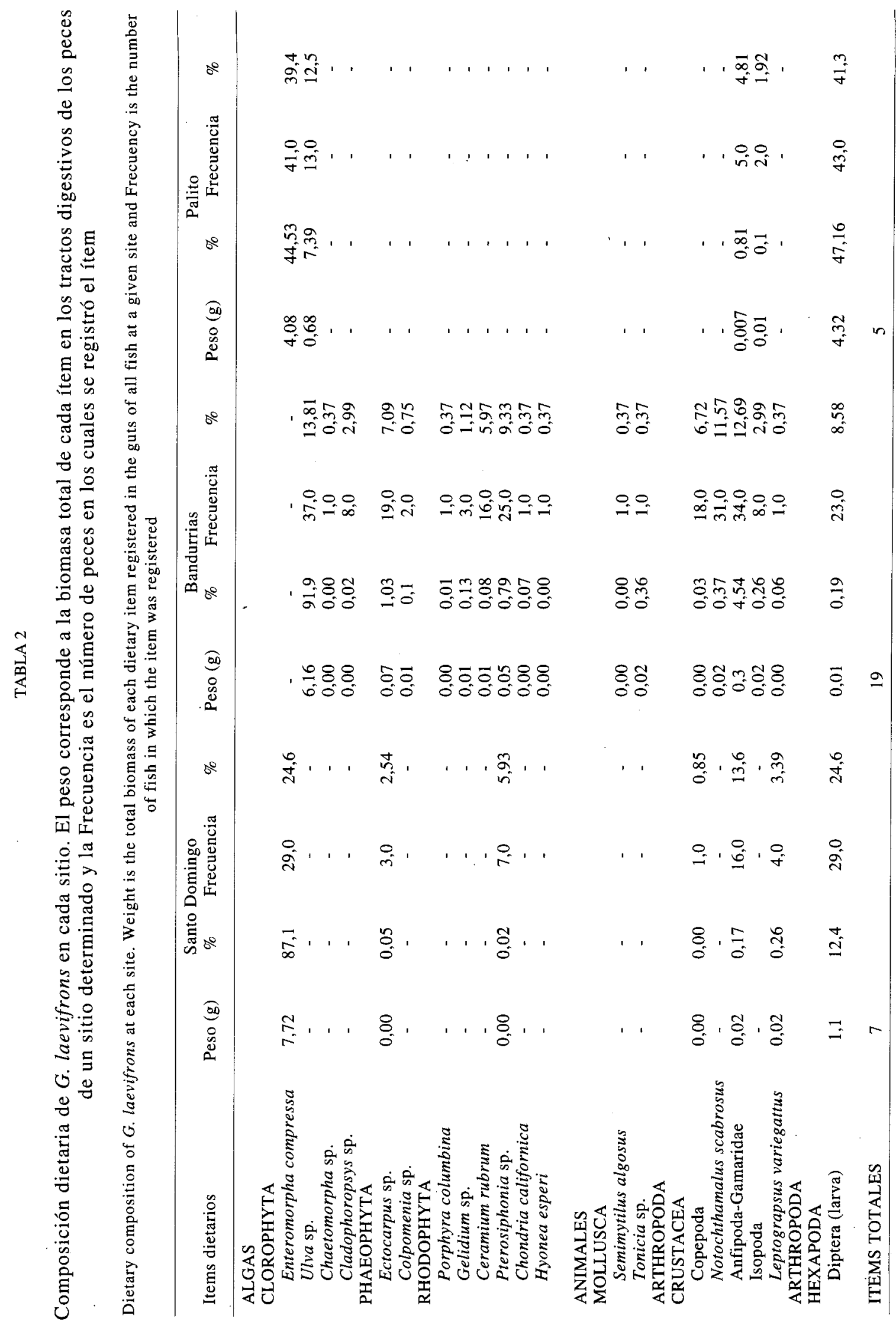


ítems presa (19 ítems), mientras que en Santo Domingo y Palito la dieta estuvo compuesta por un reducido número de ítems ( 7 y 5 respectivamente). Si bien en todas las localidades los ítems presa más importantes fueron las algas verdes ( $E$. compressa y Ulva sp.) y los artrópodos (Anfípodos-Gamáridos, Notochthamalus scabrosus y larvas de dípteros), en Bandurrias se observó una composición dietaria diferente de la de Santo Domingo y Palito, los cuales no mostraron diferencias entre sí. Estas diferencias y similitudes se expresaron en la correlación significativa observada sólo entre los rangos dietarios de Palito y
Santo Domingo $\left(r_{s}=0,46\right.$ y 0,48 con $P=0,03$ y $P$ $=0,02$, para los rangos de peso y frecuencia de ocurrencia respectivamente). Cobertura de especies sésiles en las pozas inter-
mareales

En los 90 cuadrantes fotográficos analizados se identificó a 5 ítems principales (Fig. 2a): roca desnuda, E. compressa, Ulva sp., algas rojas incrustantes (Lithotamiales) y Collisella araucana. En Santo Domingo el substrato estuvo dominado
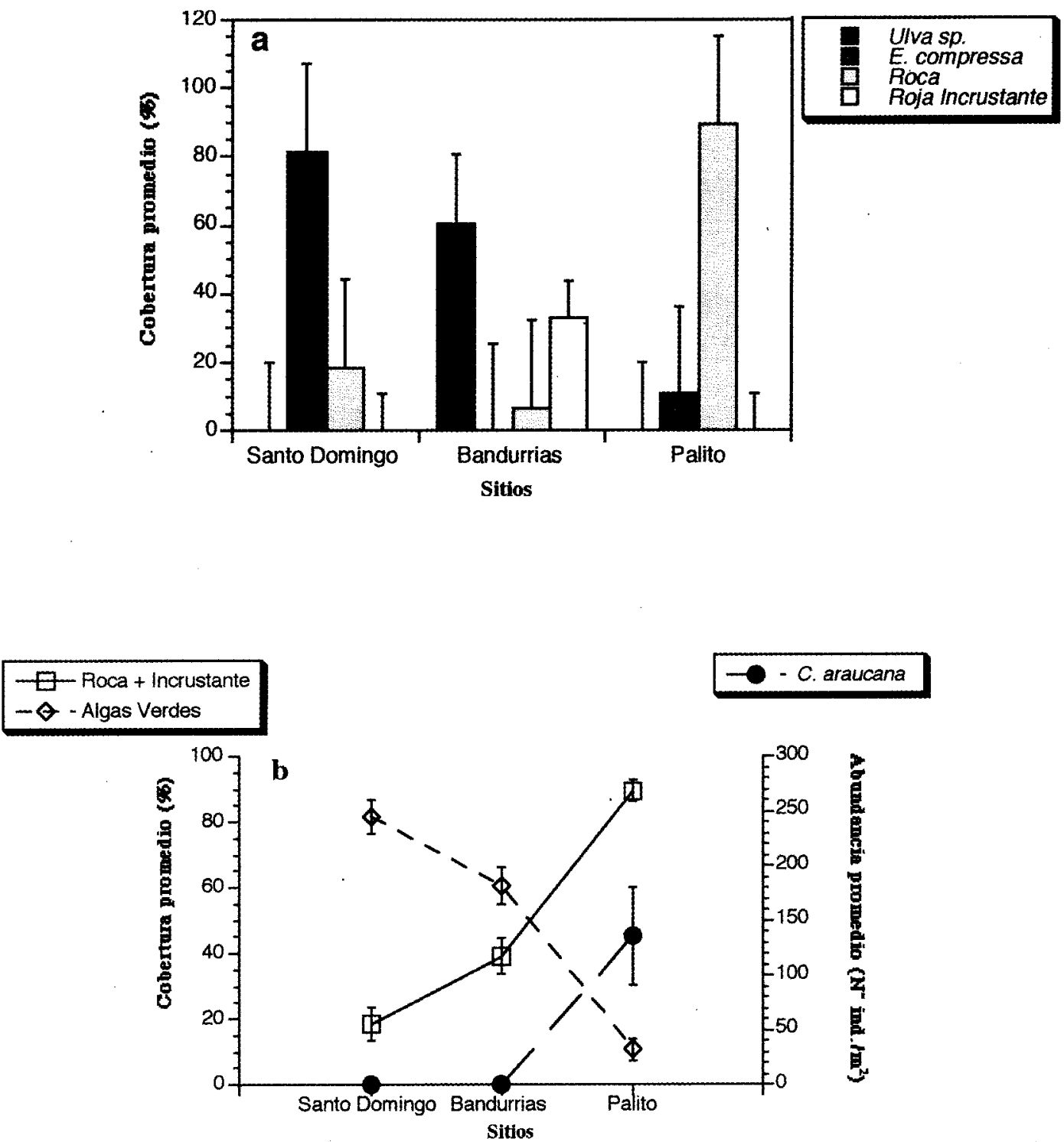

Fig. 2. Coberturas y abundancias promedio $( \pm 1 \mathrm{DE})$ de ítems sésiles en las paredes y fondo de las pozas intermareales muestreadas. a: Cobertura promedio de los principales ítems en cada sitio. b: Cobertura promedio de ítems categorizados y abundancia de $C$. araucana en cada sitio.

Mean cover and abundance $( \pm 1$ SD) of sesile items on the walls and bottom of the sampled intertidal pools. a: Mean cover of the main ítems at each site. $b$ : Mean cover of the categorized items and abundance of $C$. araucana at each site. 
por E. compressa con una cobertura promedio de $81,4 \%$. En Bandurrias los ítems con mayores porcentaje de cobertura promedio fueron Ulva sp. $(60,6 \%)$ y las algas rojas incrustantes $(32,9 \%)$. Ambos ítems no fueron observados en Santo Domingo ni en Palito. En Palito el ítem dominante fue roca desnuda $(89,5 \%)$ y se observó una baja cobertura de $E$. compressa $(10,6 \%)$. Además, en esta localidad se observaron altas densidades promedio de Collisella araucana (135 individuos/ $\mathrm{m}^{2}$ ), los cuales no fueron observados en el resto de las localidades

Tomando en cuenta la composición dietaria de G. laevifrons y el hecho de que los ítems sésiles que son dominantes en un sitio están prácticamente ausentes en el resto, para realizar el análisis comparativo de la oferta de presas en cada localidad se consideró a las coberturas de $E$. compressa y Ulva sp. en conjunto bajo la categoría de Algas Verdes. La cobertura promedio de Algas Verdes (Fig. 2b) mostró diferencias significativas entre las tres localidades $\left(\mathrm{F}_{0,05 ; 2,87}=1472\right.$; $\mathbf{P}<0,0001)$ siguiendo la secuencia de Santo Domingo $(81,4)>$ Bandurrias $(60,6)>$ Palito $(10,6)$. Asimismo, los ítems sésiles que no aparecen representados en la dieta de $G$. laevifrons tales como la roca desnuda y las algas rojas incrustantes, fueron agrupados bajo la categoría de Roca + Incrustantes. La cobertura promedio de esta categoría (Fig. 2b) mostró diferencias significativas entre las localidades $\left(\mathrm{F}_{0,05: 2,87}=1472 ; \mathrm{P}<0,0001\right)$, siguiendo la secuencia de Palito $(89,5)>$ Bandurrias $(39,2)>$ Santo Domingo $(18,5)$. Finalmente, Collisella araucana sólo fue observada en Palito (Fig. 2b).

\section{Tamaños y biomasas corporales}

El tamaño (Fig. 3a) y peso (Fig. 4a) de los ejemplares capturados en Santo Domingo fluctuó entre $\operatorname{los} 81$ a $148 \mathrm{~mm}$ y entre $\operatorname{los} 8,1$ a $60,3 \mathrm{~g}$, respectivamente. El tamaño (Fig. 3 b) y peso (Fig. 4b) de los ejemplares capturados en Bandurrias fluctuó entre los 60 a $122 \mathrm{~mm}$ y entre $\operatorname{los} 3,0$ a 49,4 g respectivamente. En Palito el tamaño (Fig. 3c) y peso (Fig. 4c) de los ejemplares capturados fluctuó entre los 49 a $99 \mathrm{~mm}$ y entre los 1,3 a 18,1 g. Tanto los promedios de tamaño corporal y peso mostraron diferencias entre los sitios $\left(\mathrm{F}_{0,05: 2,107}=\right.$ $56 ; \mathrm{P}<0,0001$ y $\mathrm{F}_{0,05 ; 2,107}=62 ; \mathrm{P}<0,0001$ para tamaño y peso respectivamente). La secuencia de diferencias significativas entre los sitios fue: Santo Domingo (112 mm y $245 \mathrm{~g})>$ Bandurrias $(81 \mathrm{~mm}$ y $9,9 \mathrm{~g})>$ Palito (67 $\mathrm{mm}$ y $5,4 \mathrm{~g}$ ).

En cada localidad (Fig. 5) se observó una relación positiva y significativa entre el tamaño cor- poral y el peso de los ejemplares capturados $\left(\mathrm{F}_{0,05: 1,28}=379 ; \mathrm{P}<0,001 ; \mathrm{F}_{0,05 ; 1,35}=2335 ; \mathrm{P}<\right.$ 0,0001 y $\mathrm{F}_{0,05 ; 1,41}=584 ; \mathrm{P}<0,0001$ para Santo Domingo, Bandurrias y Palito, respectivamente). La pendiente de esta relación fue significativamente diferente entre los sitios $\left(F_{0,05 ; 2,1,2,104}=5,9\right.$ : $P=0,0036)$. La secuencia de diferencias significativas fue: Palito $>$ Bandurrias $>$ Santo Domingo. Debido a las diferencias significativas de los tamaños corporales de cada sitio, el análisis de homogenidad de pendientes se realizó basándose
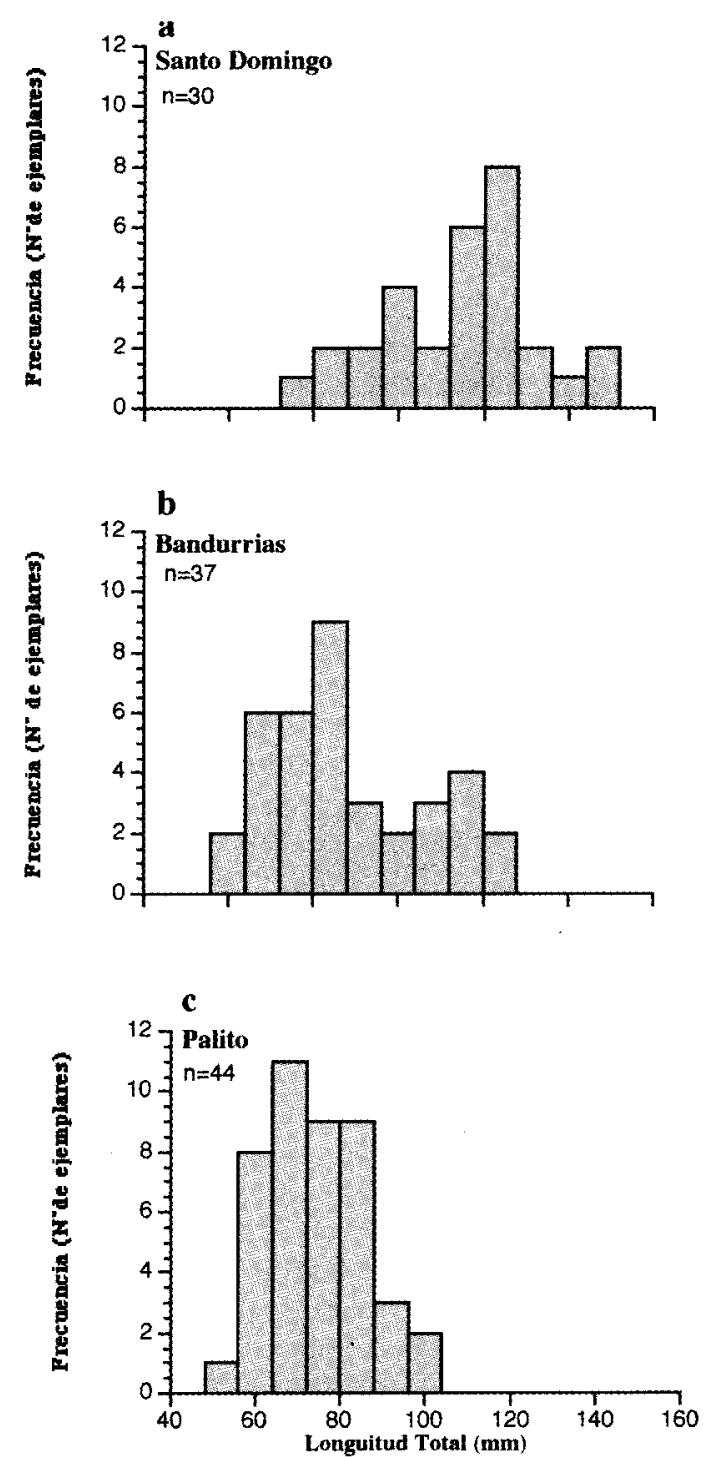

Fig. 3. Histogramas del tamaño corporal de los ejemplares de $G$. laevifrons capturados en cada sitio: a: Santo Domingo, b: Bandurrias y c: Palito.

Body sizes histograms of the individual of $G$. laevifrons caugth in each site: $a$ : Santo Domingo, b: Bandurrias and $c$ : Palito. 
en la interacción entre el factor sitio y la covariable peso, en un modelo fijo de Análisis de Varianza de una vía (Huitema 1980).

Concentración de metales pesados en el agua de mar y tejidos

Los análisis de la concentración de metales pesados en el agua de mar (Fig. 6a) muestran que, de los tres metales analizados, sólo el cobre presentó

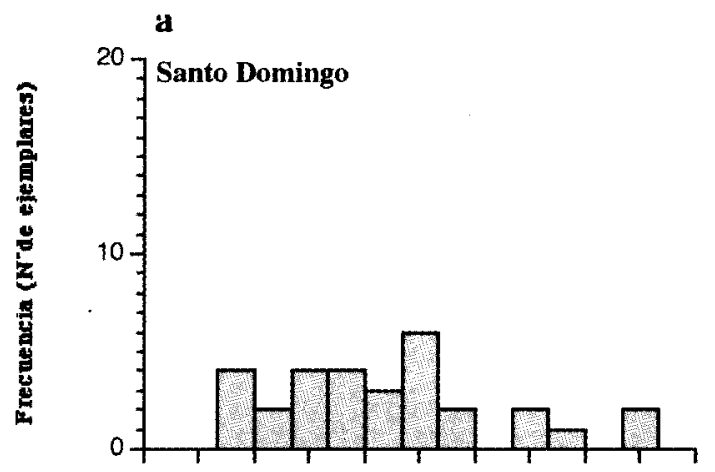

b

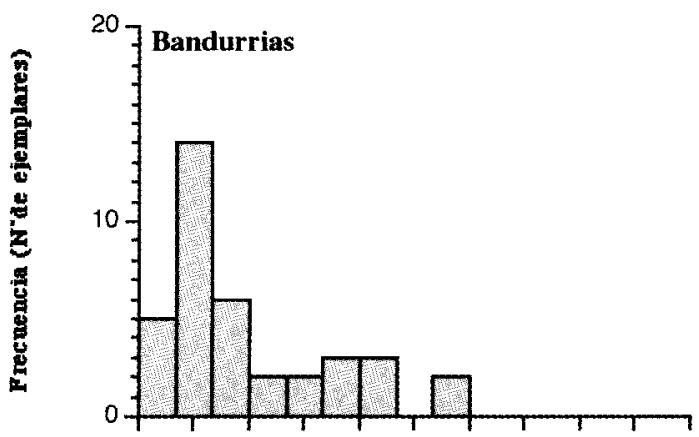

c

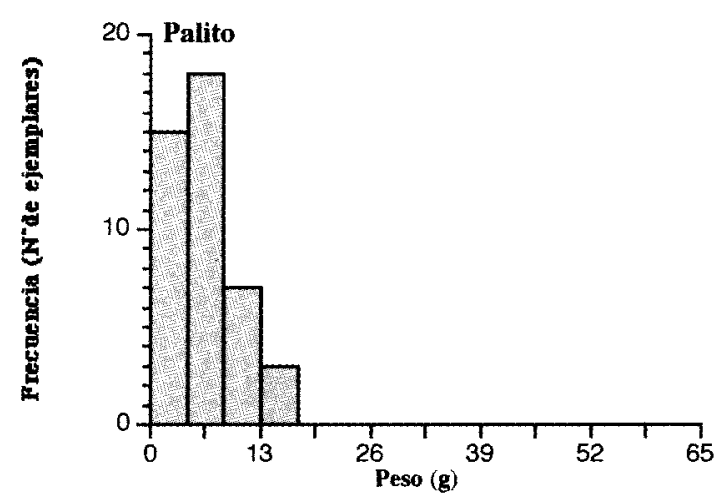

Fig. 4. Histogramas del peso corporal de los ejemplares de $G$. laevifrons capturados en cada sitio: a: Santo Domingo, b: Bandurrias y c: Palito.

Body weight histograms of the individual of G. laevifrons caugth in each site: $a$ : Santo Domingo, b: Bandurrias and c: Palito. diferencias estadísticas entre sitios $\left(\mathrm{F}_{0,05 ; 2,15}=408\right.$; $\mathrm{P}<0,0001 ; \mathrm{F}_{0,05 ; 2,15}=0,58 ; \mathrm{P}=0,57 ; \mathrm{F}_{0,05 ; 2,15}=1 ; \mathrm{P}$ $=0,39$, para cobre, zinc y cadmio respectivamente). La concentración de este metal en Palito y Santo Domingo superó en más de un orden de magnitud a la de Bandurrias. La secuencia de diferencias significativas entre las concentraciones promedio de este metal en los sitios fue: Palito $(31,3 \mu \mathrm{g} / \mathrm{l})>$ Santo Domingo $(27,1 \mu \mathrm{g} / \mathrm{l})>$ Bandurrias $(2,3 \mu \mathrm{g} / 1)$.
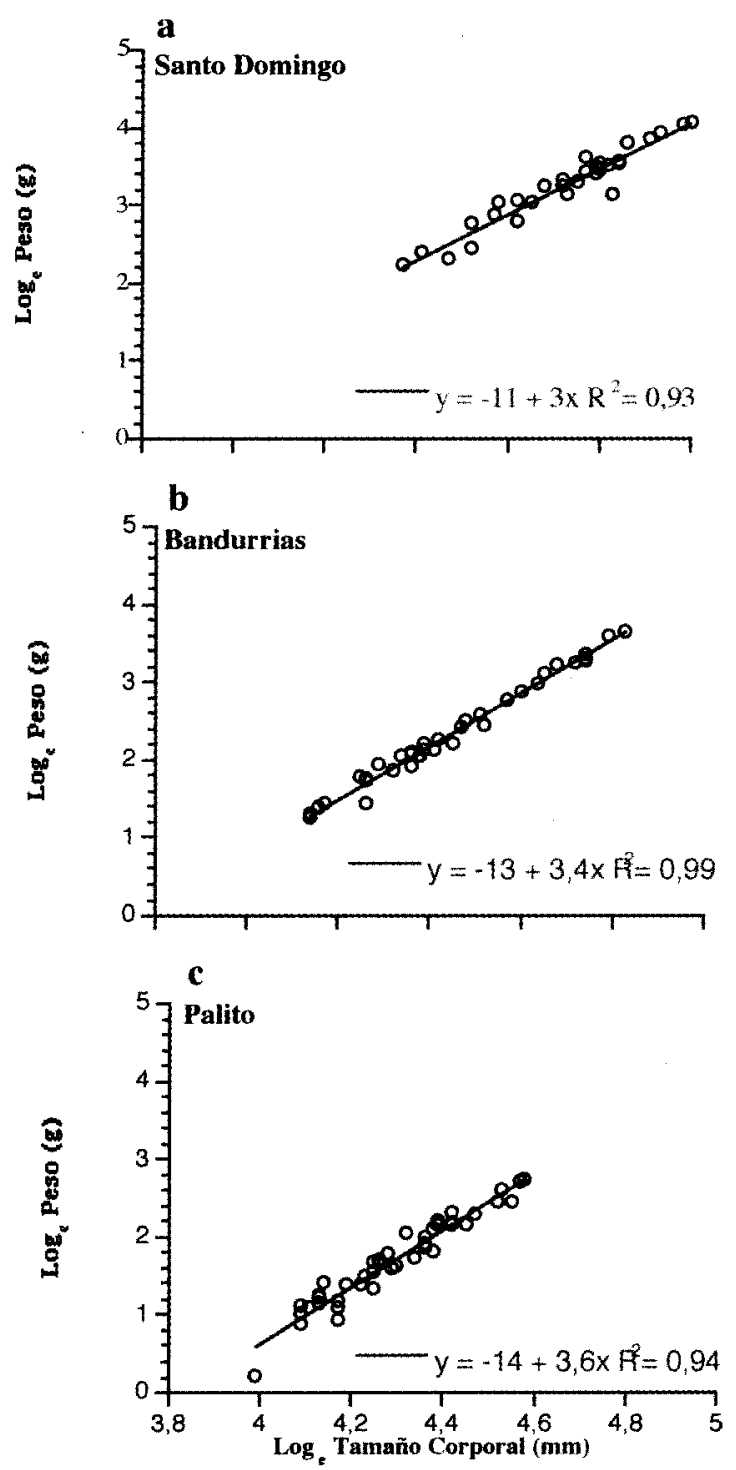

Fig. 5. Relaciones entre el tamaño corporal y el peso de los ejemplares de $G$. laevifrons capturados en cada sitio: a: Santo Domingo, b: Bandurrias y c: Palito.

Relationships between body size and weight of the individuals of G. laevifrons caught in each site: a: Santo Domingo, b: Bandurrias and c: Palito. 
Tanto en Santo Domingo como en Palito se observaron altas concentraciones de cobre en los tejidos de E. compressa. En estas mismas localidades, la concentración de cobre en los tejidos de G. laevifrons también fue alta (Fig. 6b). Las diferencias significativas en la concentración de cobre en los tejidos de las algas verdes siguieron la
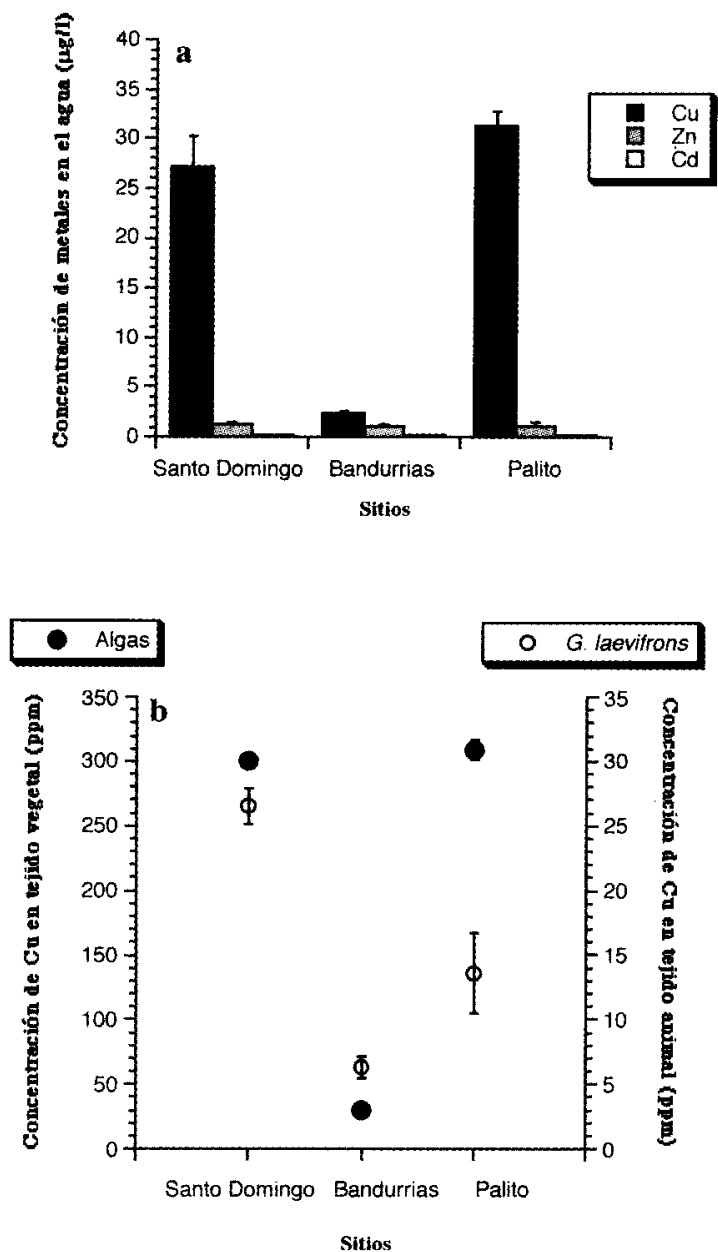

Fig. 6. Concentración promedio $( \pm 1 \mathrm{DE}) \mathrm{de}$ metales en el agua de mar y cobre en tejidos de los organismos muestreados en cada sitio. a: Concentración de metales pesados en el agua de mar de cada sitio. b: Concentración de cobre en el tejido vegetal de las algas (eje y 1) y en el tejido animal de $G$. laevifrons (eje y 2 ) muestreados en cada sitio.

Mean concentration $s( \pm 1$ SE) of trace metals in the sea water and copper in the tissues of the organisms sampled at each site. a: Trace metal in the sea water at ecah site. b: Copper concentration in the algal tissues (y axis 1 ) and in the animal tissues of $G$. laevifrons (y axis 2 ) sampled at each site. secuencia: Palito $(308 \mathrm{ppm})>$ Santo Domingo $(300 \mathrm{ppm})>$ Bandurrias $(30 \mathrm{ppm})$. En el caso de la concentración de cobre en los tejidos de $G$. laevifrons la secuencia fue: Santo Domingo (26 ppm) > Palito (13 ppm) > Bandurrias $(6 \mathrm{ppm})$.

\section{DISCUSION}

\section{Análisis dietario}

La composición trófica mostró diferencias entre los sitios contaminados (Santo Domingo y Palito) y no contaminados (Bandurrias), las cuales se relacionan con la diversidad de ítems presa disponibles en la zona intermareal (ver Fariña $1998^{3}$ y Tabla 1). Sin embargo, las algas verdes fueron uno de los ítems presa más consumidos en ambos tipos de sitios. La preferencia por las algas verdes ha sido descrita para $G$. laevifrons en la zona central de Chile, y estaría relacionada con su buena calidad como alimento y su alta digestibilidad (CW Cáceres, comunicación personal). Los artrópodos fueron otro grupo de presas de alta representación en ambos tipos de sitios. En los sitios contaminados este grupo estuvo representado principalmente por las larvas de dípteros, mientras que en el no contaminado estuvo representado por cirripedios y anfípodos. La alta representación de las larvas de dípteros en los sitios contaminados podría estar relacionada con la dominancia de algas efímeras (como E. compressa) en estos sitios. Robles \& Cubit (1981), trabajando en el intermareal rocoso de California, demostraron que luego de la ocurrencia de tormentas el substrato rocoso es monopolizado por especies de algas efímeras (principalmente del género Enteromorpha), y que esta situación favorece el crecimiento de abundantes poblaciones de dípteros que consumen a las algas.

\section{Cobertura de especies sésiles en las pozas inter- mareales}

Los resultados de los análisis de cobertura de especies sésiles presentes en las paredes y fondo de las pozas intermareales concuerdan con los patrones de diversidad observadas en plataformas intermareales de la costa norte de Chile efectadas y no afectadas por la contaminación derivada de la minería del cobre (Castilla 1995). En relación con la dieta de G. laevifrons, se observaron im-

\footnotetext{
${ }^{3}$ Fariña JM (1998) Conducta trófica de tres especies intermareales del norte de Chile en relación con la presencia de contaminantes derivados de la minería del cobre. XLI Reunión Anual de la Sociedad de Biología de Chile, Pucon, Chile: 93.
} 
portantes diferencias en la disponibilidad de algas verdes de los tres sitios de estudio La cobertura promedio de E. compressa en Santo Domingo fue mayor que la cobertura de Ulva sp. en Bandurias, mientras que Palito presentó el menor promedio de cobertura de algas verdes de las tres localidades. En Palito, se observó a E. compressa sólo sobre las conchas de los "patelogastrópodos" herbívoros Collisella araucana, constatándose además un alto porcentaje de roca desnuda. Históricamente, sólo en los últimos años Collisella araucana ha invadido la zona intermareal rocosa de Palito, y sería responsable de la eliminación o disminución de $E$. compressa (JC Castilla, comunicación personal). La presencia de Collisella araucana no fue constatada en Santo Domingo, mientras que en Bandurrias estos organismos se encontraron en bajas densidades en las pozas de la zona intermareal alta.

\section{Tamaños y biomasas corporales}

Los tamaños corporales y pesos, al igual que las relaciones alométricas entre tamaño corporal y peso de los ejemplares capturados, mostraron diferencias significativas entre los tres sitios de estudio. Los ejemplares colectados en Santo Domingo fueron de mayor tamaño que los colectados en Bandurrias y estos, fueron de mayor tamaño que los colectados en Palito. Hasta el momento, no se han desarrollado estudios en nuestro país que permitan determinar el rango de variación espacial de estos atributos en los peces intermareales como grupo, o en $G$. laevifrons en particular.

En relación con estas diferencias, se ha descrito que G. laevifrons utiliza la zona intermareal sólo en su etapa juvenil, migrando hacia zonas submareales cuando alcanza tamaños corporales mayores (Stepien 1990, Pulgar et al. 1999), y que la utilización de la zona intermareal en esta especie estaría condicionada por la disponibilidad de alimento (Varas \& Ojeda 1990, Pulgar et al. 1999). Bajo esta perspectiva, seria esperable de que el tiempo de residencia de esta especie en la zona intermareal estuviese determinado por la disponibilidad relativa de alimento. De esta forma en los sitios con alta disponibilidad de alimento los tiempos de residencia serían mayores y por ende se observarían tamaños corporales mayores que los encontrados en sitios con baja disponibilidad de alimento. Los resultados del presente estudio entregan evidencia sugerente para este perspectiva.
En el sitio con mayor cobertura de algas verdes (Santo Domingo) se observaron los tamaños corporales mayores y en el sitio con menor cobertura de algas (Palito) se observaron los tamaños corporales menores.

Concentración de metales pesados en el agua de mar y tejidos

Los análisis de la concentración de metales pesados, y particularmente cobre, dan cuenta de que tanto Santo Domingo como Palito corresponden a sitios afectados por contaminantes derivados de la minería del cobre. Una serie de trabajos desarrollados tanto en la zona de Chañaral como en otras localidades afectadas por la contaminación derivada del cobre, reportan concentraciones similares a las observadas en este estudio (ver Castilla \& Nealler 1981, Castilla 1995, Fariña \& Castilla 19984, Vásquez et al. 1999).

En los sitios contaminados, las altas concentraciones de cobre en E. compressa, siguen la secuencia de las concentraciones observadas en el agua de mar, y concuerdan con resultados de trabajos anteriores desarrollados en las cercanías de Chañaral (Castilla \& Correa 1997). Las diferencias entre las concentraciones de metales pesados en los organismos presa (algas) y los consumidores ( $G$. laevifrons) siguen el patrón de transferencia (de un $10 \%$ de concentración intra tejidos) entre niveles tróficos contiguos (Cabana \& Rasmussen 1994). Sin embargo, las concentraciones en los tejidos de $G$. laevifrons no siguen la misma secuencia de diferencias entre sitios (los ejemplares de Santo Domingo presentan mayores concentraciones en sus tejidos que los de Palito). Estas diferencias podrían ser explicadas por una menor tasa de consumo de $E$. compressa por parte de G. laevifrons (ver Tabla 2) o por una menor disponibilidad de esta alga en el sitio de Palito.

En el presente estudio se ha demostrado que los ejemplares de $G$. laevifrons presentes en zonas del intermareal rocoso del norte de Chile presentan diferencias en su composición dietaria, la cual se relaciona con las diferencias en composición y abundancia de los ensambles de organismos sésiles presentes en sitios afectados y no afectados por contaminantes derivados de la minería del cobre. Por otra parte, se sugiere que las diferencias en los tamaños corporales y el traspaso a través de la dieta de metales pesados pueden estar relacionadas con la disponibilidad de alimento, la cual es

\footnotetext{
${ }^{4}$ Fariña JM \& JC Castilla (1998) Top-down and pushing-up: when the ephemeral becomes ethernal, the consumers are consumed, and the lizards go to the beach. Mellon Workshop 98, Las Cruces, Chile: 2.
} 
dependiente de la presencia o ausencia de otros organismos herbívoros intermareales (i.e., patelogastrópodos herbívoros).

\section{AGRADECIMIENTOS}

Se agradece a los taltalinos: Maximino, Heinz, Esau, Jorge Erazo y familia; y a los santiaguinos: José Miguel Rojas, Andrea Angel y Anna Astorga por su inapreciable ayuda y colaboración. Especiales agradecimientos a Plan 9 y constant concept. Este estudio fue financiado por los proyectos Fondecyt 2970075 y 4990021 a JMF y parcialmente por FONDAP O\&BM N $\mathrm{N}^{\circ} 3$ a FPO.

\section{LITERATURA CITADA}

CABANA G \& JB RASMUNSSEN (1994) Modeling food chain structure and contaminant bioaccumulation using stable nitrogen isotopes. Nature 372: 255-257.

CASTILLA JC (1975) Problemática general de la contaminación marina en Chile: evaluación, estudios y perspectivas, Primer Congreso Iberoamericano del Medio Ambiente, Centro de Estudios del Medio Ambiente, 12-18 Octubre, Madrid, España: 724-748.

CASTILLA JC (1995) Copper mine tailing disposal in northern Chile rocky shores: Enteromorpha compressa (Chlorophyta) as a sentinel species. Environmental Monitoring and Assessment 40: 171-184.

CASTILLA JC \& JA CORREA (1997) Copper tailing impacts in coastal ecosystems of northern Chile: from species to community responses. National Health Forum Monographs, Metal Series 3: 81-92.

CASTILlA JC \& E NEALLER (1978) Marine environmental impact due to mining activities of El Salvador Copper Mine, Chile. Marine Pollution Bulletin 9: 67-70.

CORREA JA, P GONZALEZ, P SANCHEZ, P MUÑOZ \& MC ORELLANA (1996) Copper-algae interactions: inheritance or adaptation? Environmental Monitoring and Assessment, 39: 41-54
HUITEMA BE (1980) The analysis of covariance and alternatives. John Wiley \& Sons, New York, USA. $442 \mathrm{pp}$.

INGENIERIA Y GEOTECNICA LTDA (1990) Levantamiento catastral de los tranques de relaves en Chile. Servicio Nacional de Geología y Minería, Santiago, Chile. $1200 \mathrm{pp}$.

MUÑOZ AA \& FP OJEDA (1997) Feeding guild structure on a rocky intertidal fish assemblage in central Chile. Environmental Biology of Fishes 49: 471-479.

PULGAR J, F BOZINOVIC \& FP OJEDA (1999) Behavioral thermoregulation in the intertidal fish Girella laevifrons (Kyphosidae): the effect of starvation. Marine and Freshwater Behaviour and Physiology 32: 27-38.

ROBLES C \& J CUBIT (1981) Influence of biotic factors in an upper intertidal community: Dipterean larvae grazing on algae. Ecology 62: 1536-1547.

SIEGEL S \& NJ CASTELLAN (1988) Nonparametric statistics for the behavioral sciences. McGraw-Hill, New York, USA. 399 pp.

STEPIEN CA (1990) Population structure, diets and biogeographic relationships of a rocky intertidal fish assemblage in central Chile. High levels of herbivory in a temperate system. Bulletin of Marine Science 47: 598-612.

UNDERWOOD AJ (1997) Experiments in Ecology. Cambridge University Press, Melbourne, Australia. $504 \mathrm{pp}$.

VARAS E \& FP OJEDA (1990) Intertidal fish assemblage of the central Chilean coast: diversity, abundance and trophic patterns. Revista de Biología Marina (Valparaiso) 25: 59-70.

VASQUEZ JA, JMA VEGA, B MATSUHIRO \& C URZUA (en prensa) The ecological effects of minning discharges on subtidal habitats dominated by macroalgae in northern Chile: population and community level. Hydrobiologia.

WILKINSON L, G BLANK \& C GRUBER (1996) Desktop data analysis with SYSTAT. Prentice Hall, New Yersey, USA. 798 pp. 